\title{
Re-evaluation of Need for Bone Marrow Examination in Patients with Isolated Thrombocytopenia Contributors
}

\author{
Abhishek Purohit ${ }^{1} \cdot$ Mukul Aggarwal $^{1} \cdot$ Pawan Kumar Singh $^{1} \cdot$ \\ Manoranjan Mahapatra ${ }^{1} \cdot$ Tulika Seth $^{1} \cdot$ Seema Tyagi $^{1}$. \\ Renu Saxena $^{1} \cdot$ Hara P. Pati ${ }^{1} \cdot$ Pravas Mishra $^{1}$
}

Received: 9 May 2014/ Accepted: 19 March 2015/Published online: 28 March 2015

(C) Indian Society of Haematology \& Transfusion Medicine 2015

\begin{abstract}
Diagnosis of immune thrombocytopenia (ITP) is based on clinical suspicion and normal peripheral smear except for thrombocytopenia. Bone marrow examination is carried out to rule out leukemia, myelodysplastic syndrome or aplastic anemia. However, in most cases, clinical diagnosis is not altered after the bone marrow reports. Hence, this present study was carried out to evaluate the justification for bone marrow examination in the setting of isolated thrombocytopenia. All patients presenting to the hematology OPD with isolated thrombocytopenia and suspected diagnosis of ITP, between October 2011 and April 2013, were included in the study. Data was collected from bone marrow reports and outpatient records. A total
\end{abstract}

Pravas Mishra

pravas_mishra@rediffmail.com

Abhishek Purohit

purohitabhi80@gmail.com

Mukul Aggarwal

mukulmamc@gmail.com

Pawan Kumar Singh

pawan2809@gmail.com

Manoranjan Mahapatra

mrmahapatra@hotmail.com

Tulika Seth

tuliseth@yahoo.com

Seema Tyagi

drseematyagi@hotmail.com

Renu Saxena

renusaxena@outlook.com

Hara P. Pati

harappati@yahoo.com

1 Department of Hematology, All India Institute of Medical Sciences, New Delhi, India of 353 cases were found. 319 cases had features of typical ITP and the rest had some form of organomegaly and/or lymphadenopathy. Bone marrow examination in all cases revealed normal hematopoietic elements and prominence of megakaryocytes including juvenile forms with no novel diagnosis in any patient. Routine use of bone marrow examination in the diagnostic workup of isolated thrombocytopenia is not required in our center even if steroids are planned as a first line therapy. However, a detailed history, thorough examination with complete hemogram and peripheral smear examination are essential.

Keywords Immune thrombocytopenia $\cdot$ Bone marrow examination

\section{Introduction}

Immune thrombocytopenia (ITP) is a diagnosis of exclusion. It is characterized by immune-mediated accelerated platelet destruction and suppressed platelet production. ITP presents as primary (isolated) ITP or as secondary ITP in the context of other diseases such as autoimmune disorders and infections. Diagnosis is based on clinical suspicion and a normal peripheral smear except for thrombocytopenia. However, bone marrow examination is carried out in many cases to rule out leukemia, myelodysplastic syndrome or aplastic anemia. Consensus guidelines do not recommend a bone marrow examination in cases with isolated thrombocytopenia [1] even when steroids are considered as first line therapy (grade $2 \mathrm{C}$ recommendation).

The prevalent practice at our centre has been to perform a bone marrow examination in all patients suspected of ITP, whenever steroids are considered as first line therapy. This study was carried out to re-evaluate this practice. 


\section{Materials and Methods}

All patients presenting to the Hematology OPD with a suspected diagnosis of ITP were analysed retrospectively. Data was collected from bone marrow requisition forms and outpatient records. A typical patient attending our OPD would have undergone a detailed workup including history, physical, complete blood count and peripheral smear examination before a bone marrow examination was requisited. A bone marrow examination was waived if the patient were to receive immunoglobulins as first line therapy. All bone marrow requisition forms in our laboratory were screened to detect cases where ITP was suspected. The clinical history and other details entered in the requisitions were confirmed by going through the corresponding case files in our OPD. These case files also provided data on follow up complaints, history, physical examinations, treatment details and outcome. Cases were categorized according to age, sex, signs and symptoms as well as duration of illness. Based on duration of symptoms, cases were divided into three groups, those with symptoms of less than 3 months duration (acute/newly diagnosed ITP), those with symptoms of more than 12 months duration (chronic ITP), and those with 3-12 months (persistent ITP) duration of symptoms.

\section{Results}

We retrieved 353 cases from the clinical records. All cases had undergone a bone marrow examination. Of 353 cases, $178(50.4 \%)$ were females. Based on age, cases were categorized into six groups as shown in the Table 1. Only one patient was less than a year of age in this cohort (six month old female child).

Patients with ITP presented with various bleeding symptoms (Table 1). Severe life threatening bleeds including gastrointestinal bleed, hematuria or intracranial bleed were uncommon.

Of all cases, 34 patients (age range 0.5-78 years) presented with organomegaly (hepatomegaly, splenomegaly) and/or lymphadenopathy (Table 1). The maximum extent of either hepatomegaly or splenomegaly was $2 \mathrm{~cm}$ below the costal margin. None of the reported cases with baseline cervical lymph nodes had nodes any larger than approximately $0.5 \mathrm{~cm}$. Subsequent case notes on followup did not record any further change in baseline findings. None of these cases, on followup (median follow up 14 months; range 16-34 months), have developed any other findings to suggest an alternate diagnosis.

Besides bleeding, 53 patients also had other constitutional symptoms in their clinical presentation. 24 (6.8\%)
Table 1 Presentation of ITP cases

\begin{tabular}{lc}
\hline & Number of patients $(\%)$ \\
\hline Age wise distribution of ITP cases & \\
Age & \\
Less than five years & $40(11.3)$ \\
$5-12$ & $47(13.3)$ \\
$13-18$ & $48(13.6)$ \\
$19-40$ & $143(40.51)$ \\
$41-60$ & $61(17.28)$ \\
More than 60 years & $14(3.9)$ \\
Clinical bleeding symptoms and their relative frequency \\
Bleeding symptoms \\
Petechiae & $268(75.92)$ \\
Epistaxis & $116(32.86)$ \\
Gum bleeding & $126(35.7)$ \\
Gastrointestinal bleeding & $21(5.97)$ \\
Hematuria & $10(2.8)$ \\
Intracranial Bleeding & $4(1.1)$ \\
Menorrhagia & $55(30.9)$ \\
Patient frequency based on duration of the symptoms \\
Duration of symptoms
\end{tabular}

patients presented with a prior history of fever and 29 $(8.2 \%)$ with weakness in addition to bleeding symptoms.

All 353 patients underwent bone marrow aspirate and biopsy examination, which revealed normal hematopoietic elements and prominence of megakaryocytes including juvenile forms in all cases. None of the cases who presented with organomegaly and/or lymphadenopathy have developed features to suggest an alternate diagnosis on followup (median follow up 14 months; range 16-34 months). 


\section{Discussion}

ITP is characterized by thrombocytopenia secondary to peripheral destruction as well as megakaryocyte changes in the bone marrow [2,3]. A shift to young, immature, less polyploid megakaryocytes and fewer mature megakaryocytes is very common. Dysplastic forms along with bare forms and micro-megakaryocytic forms are seen in half the cases [2]. However the role of bone marrow in ITP has remained doubtful when thorough clinical history and careful general physical examination has been coupled with a detailed peripheral blood smear examination. There is agreement that a complete blood cell count and examination of the peripheral blood smear are essential for the diagnosis of ITP [4].

Studies in both children and adults showed that bone marrow examination did not change the diagnosis in presence of isolated thrombocytopenia [5-8]. Caroline C et al., in their retrospective study on 322 children with typical hematologic features of ITP, concluded that the risk of missing the diagnosis of leukemia was not greater than $1 \%$ [6]. Westerman et al., in their study on adults less than 65 years age [7] noted 5 patients (of 66), who had atypical marrow picture at baseline but on follow-up did not develop any features to suggest an alternative diagnosis such as leukemia, myelodysplasia, or aplastic anemia. Klaassen $\mathrm{RJ}$ et al. constructed a decision tree for the initial management of a child older than age 6 months, presenting with idiopathic thrombocytopenia, without blasts on the peripheral smear [9]. They studied three strategies: (1) initial BMA in all patients; (2) initial BMA only in patients at high risk; and (3) empiric therapy for all patients without initial BMA. The results were expressed as quality-adjusted life years (QALYs), a measure that estimates the overall life expectancy in years for patients receiving a particular treatment strategy, corrected for the patient's quality of life. The study concluded that the initial BMA did not significantly change the overall QALYs of a child presenting with thrombocytopenia and, consequently, is not mandatory in every patient before starting steroids [9].

Mahabir et al., in their study on 32 adult ITP patients and 51 controls, concluded that overall sensitivity and specificity of bone marrow examinations were 24 and $90 \%$, respectively [10]. Conversely, in a retrospective data on 2239 children with acute lymphoblastic leukemia, no child presented with an isolated thrombocytopenia and other abnormal physical or hematological abnormalities [11].

We had earlier described two cases of leukemia that presented to our department but were diagnosed as ITP elsewhere [12]. One child with acute lymphoblastic leukemia had received steroids for suspected ITP. The other child had acute myeloid leukemia. Similar cases prompted us to follow a policy of bone marrow examination in all suspected cases of ITP, unless immunoglobulin was planned as first line therapy. Most American pediatric hematology/oncology specialists perform a bone marrow aspirate when corticosteroids are prescribed. In contrast, Australian pediatricians were less likely to examine the bone marrow if corticosteroids were prescribed [13, 14]. Bone marrow examination led to an alternate diagnosis in $3.9 \%$ of 127 children in one study [15]. However the baseline physical and laboratory features in these cases were atypical of ITP [15]. The primary lacuna in the two cases described by us [12] was the absence of a description of baseline peripheral smear. The physical examination had been done elsewhere and could not be verified.

Organomegaly in ITP is not unknown. Mitura et al. [16] described splenomegaly in as many as 48 out of 72 ITP patients, while Hijazi et al. [17] described it in 31/56 Arab children.

The majority of our patients with a diagnosis of ITP receive steroids as first line therapy. Hence we had felt justified in continuing with the policy of a bone marrow examination for all patients [12]. However an increasing burden of bone marrow examinations, quite often resulting in treatment delays till a bone marrow examination was performed, prompted us to review this policy and conduct a retrospective analysis of our data.

Being retrospective in nature, our study has usual limitations of selection bias and possibility of incomplete documentation (although we did look at follow up data from outpatient case notes to ensure complete data). Besides, only 14 patients $(3.9 \%)$ were 60 years or older where myelodysplastic syndrome could be considered. The guidelines published by the American Society of Hematology do not recommend a bone marrow examination in any age group provided a typical presentation of ITP (grade $2 \mathrm{C}$ recommendation) [1].

We conclude from this retrospective data that the routine use of bone marrow examination in the diagnostic workup of isolated thrombocytopenia is not required in our centre even if steroids are considered as first line therapy. We will continue to rely upon a thorough examination to rule out organomegaly and/or lymphadenopathy along with complete hemogram and peripheral smear examination. We currently would not routinely advise a bone marrow examination in children of any age with a typical presentation.

This is not a general recommendation. We are aware that some physicians might not have access to a reliable peripheral smear examination. Therefore each center would need to evaluate their own data, preferably in a prospective manner. 


\section{References}

1. Neunert C, Lim W, Crowther M et al (2011) The American Society of Hematology 2011 evidence-based practice guideline for immune thrombocytopenia. Blood 117(16):4190-4207

2. Bhasin TS, Sharma S, Manjari M, Mannan R, Kansal V, Chandey M, Piplani S (2013) Changes in megakaryocytes in cases of thrombocytopenia: bone marrow aspiration and biopsy analysis. J Clin Diagn Res 7(3):473-479

3. Deka L, Gupta S, Gupta R, Pant L, Kaur CJ, Singh S (2013) Morphometric evaluation of megakaryocytes in bone marrow aspirates of immune-mediated thrombocytopenic purpura. Platelets 24(2):113-117

4. George JN, Woolf SH, Kaskob GE et al (1996) Idopathic thrombocytopenic purpura: a practice guideline developed by explicit methods for the American Society of Hematology. Blood $88: 3$

5. Jones EC, Boyko WJ (1985) Diagnostic value of bone marrow examination in isolated thrombocytopenia. Am J Clin Pathol 84(5):665

6. Calpin C, Dick P, Poon A, Feldman W (1998) Is bone marrow aspiration needed in acute childhood idiopathic thrombocytopenic purpura to rule out leukemia? Arch Pediatr Adolesc Med 152(4):345-347

7. Westerman DA, Grigg AP (1999) The diagnosis of idiopathic thrombocytopenia purpura in adults: does bone marrow biopsy have a place? Med J Aust 170:216

8. Mak YK, Yu PH, Chan CH, Chi C (2000) The management of isolated thrombocytopenia in Chinese adults: does bone marrow examination have a role at presentation? Clin Lab Haematol 22:355

9. Klaassen RJ, Doyle JJ, Krahn MD, Blanchette VS, Naglie G (2001) Initial bone marrow aspiration in childhood idiopathic thrombocytopenia: decision analysis. J Pediatr Hematol Oncol 23(8):511-518

10. Mahabir VK, Ross C, Popovic S, Sur ML, Bourgeois J, Lim W, George JN, Wang G, Cook RJ, Toltl LJ, Nazi I, Kelton JG, Arnold DM (2013) A blinded study of bone marrow examinations in patients with primary immune thrombocytopenia. Eur J Haematol 90:121-126

11. Dubansky AS, Boyett JM, Falletta J, Mahoney DH, Land VJ, Pullen J, Buchanan G (1989) Isolated thrombocytopenia in children with acute lymphoblastic leukemia: a rare event in a pediatric oncology group study. Pediatrics 84(6):1068-1071

12. Naithani R, Kumar R, Mahapatra M, Agrawal N, Pati HP, Choudhry VP (2007) Is it safe to avoid bone marrow examination in suspected ITP? Pediatr Hematol Oncol 24(3):205-207

13. Vesely S, Buchanan GR, Cohen A, Raskob G, George J (2000) Self- reported diagnostic and management strategies in childhood idiopathic thrombocytopenic purpura: results of survey of practicing pediatric hematology/oncology specialists. J Pediatr Hematol Oncol 22(1):55-61

14. Marks M, Vadamalayan B, Ekert H, South M (2005) Intended management of children with acute idiopathic thrombocytopenic purpura: a national survey. J Paediatr Child Health 41:52-55

15. Halperin DS, Doyle JJ (1988) Is bone marrow examination justified in idiopathic thrombocytopenic purpura? Am J Dis Child 142:508-511

16. Mitura-Lesiak M, Filiks-Litwin B, Malek U, Kowalczyk JR (2004) Clinical course, diagnostic and therapeutic management of immunethrombocytopenic purpura in children. Med Wieku Rozwoj 8(4 Pt 1):1004-1011

17. Hijazi Z, Qabazard Z, Marouf R, Moosa A (1995) Splenomegaly in Arab children with idiopathic thrombocytopenic purpura. Ann Saudi Med. 15(3):209-211 